\title{
Periodic Solutions of Cohen-Grossberg-Type BAM Neural Networks with Time-Varying Delays
}

\author{
Qiming Liu', Shaoning $\mathbf{L i}^{2}$ \\ ${ }^{1}$ Institute of Applied Mathematics, Shijiazhuang Mechanical Engineering College, Shijiazhuang, China \\ ${ }^{2}$ College of Mathematics and Information Science, Shaanxi Normal University, Xi'an, China \\ Email: lqmmath@yahoo.com.cn
}

Received September 12, 2012; revised October 25, 2012; accepted November 6, 2012

\begin{abstract}
Sufficient conditions to guarantee the existence and global exponential stability of periodic solutions of a CohenGrossberg-type BAM neural network are established by suitable mathematical transformation.
\end{abstract}

Keywords: Cohen-Grossberg Neural Networks; BAM Neural Networks; Periodic Solution; Delay; Global Stability

\section{Introduction}

Many important results on the existence and global exponential stability of equilibria of neural networks with time delays have been widely investigated and successfully applied to signal processing system. However, the research of neural networks involves not only the dynamic analysis of equilibrium point but also that of periodic oscillatory solution. In practice, the dynamic behavior of periodic oscillatory solution is very important in learning theory $[1,2]$, which is motivated by the fact that learning usually requires repetition, some important results for periodic solutions of Hopfield neural networks or Cohen-Grossberg neural networks with delays have been obtained in Refs. [3-15].

The objective of this paper is to study the existence and global exponential stability of periodic solutios of a class of Cohen-Grossberg-type BAM neural networks (CGBAMNNs) with time-varying delays by suitable mathematical transformation.

The rest of this paper is organized as follows: preliminaries are given in Section 2. Sufficient conditions which guarantee the existence and global exponential stability of periodic solutions for the CGBAMNNs are established Section 3. An example is given in Section 4 to demonstrate the main results.

\section{Preliminaries}

Consider the following periodic CGNNs with time- varying delays (see Equation (1)):

For $1 \leq i \leq n, \quad t>0$ and $Z^{+}=\{1,2, \cdots, n\} . \quad x_{i}(t)$ denote the state variables of the ith neuron, $f_{j}(\cdot)$ denote the signal functions of the $j$ th neuron at time $t$; $I_{i}(t)$ denote inputs of the ith neuron at time $t ; a_{i}(\cdot)$ represent amplification functions; $b_{i}(t, \cdot)$ are appropriately behaved functions; $p_{i j}(t)$ and $u_{i j}(t)$ and are connection weights of the neural networks, respectively; $\rho_{j}$ are positive constants which correspond to the neuronal gains associated with the neuronal activations; $\tau_{i j}(t)$ correspond to the finite speed of the axonal signal transmission at time $t$ and there exist constants $\tau_{i j}$ such that $0 \leq \tau_{i j}(t) \leq \tau_{i j}, \quad p_{i j}(t), u_{i j}(t), I_{i}(t)$ and $\tau_{i j}(t)$ are all continuously periodic functions on $[0,+\infty)$ with common period $T>0$.

Throughout this paper, we assume for system (1) that

$\left(\mathrm{H}_{1}\right)$ Amplification functions $a_{i}(\cdot)$ are continuous and there exist constants $\underline{a}_{i}, \bar{a}_{i}$ such that $\underline{a}_{i} \leq a_{i}(\cdot) \leq \bar{a}_{i}$ for $1 \leq i \leq n$.

$\left(\mathrm{H}_{2}\right) \quad b_{i}(t, \cdot)$ are $T$-periodic about the first argument and there exist continuous $T$-periodic functions $\gamma_{i}(t)$ such that

$$
\begin{aligned}
& \frac{b_{i}(t, x)-b_{i}(t, y)}{x-y} \geq \gamma_{i}(t)>0, \\
& \forall x \neq y \in R, 1 \leq i \leq n
\end{aligned}
$$

$\left(\mathrm{H}_{3}\right)$ For activation functions $f_{j}(\cdot)$, there exist positive constants $L_{j}$ such that

$$
\dot{x}_{i}(t)=-a_{i}\left(x_{i}(t)\right)\left[b_{i}\left(t, x_{i}(t)\right)-\sum_{j=1}^{n} p_{i j}(t) f_{j}\left(\rho_{j} x_{j}(t)\right)-\sum_{j=1}^{n} u_{i j}(t) f_{j}\left(\rho_{j} x_{j}\left(t-\tau_{i j}(t)\right)\right)-I_{i}(t)\right]
$$




$$
L_{j}=\sup _{x \neq y}\left|\frac{f_{j}(x)-f_{j}(y)}{x-y}\right|, \forall x \neq y \in R, 1 \leq j \leq n
$$

For any continuous function $S(t)$ on $[0, T], \underline{S}$ and $\underline{S}$ denote $\min _{t \in[0, T]}\{|S(t)|\}$ and $\min _{t \in[0, T]}\{|S(t)|\}$, respectively.

For any

$$
x(t)=\left(x_{1}(t), x_{2}(t), \cdots, x_{k}(t)\right)^{\mathrm{T}} \in R^{k}, t>0,
$$

define

$$
\|x(t)\|=\sum_{i=1}^{k}\left|x_{i}(t)\right|
$$

and for any

$$
\begin{aligned}
& \varphi(s)=\left(\varphi_{1}(s), \varphi_{2}(s), \cdots, \varphi_{k}(s)\right)^{\mathrm{T}} \in R^{k}, \\
& s \in(-\sigma, 0],
\end{aligned}
$$

define

$$
\|\varphi(s)\|=\sup _{s \in[-\sigma, 0]} \sum_{i=1}^{k}\left|\varphi_{i}(s)\right|
$$

in which

$$
0<\sigma<+\infty
$$

Denote

$$
C\left([-\sigma, 0], R^{k}\right)=\left\{\psi:[-\sigma, 0] \rightarrow R^{k} \mid \psi(s)\right\}
$$

is continuous on $[-\sigma, 0]$.

Then $C\left([-\sigma, 0], R^{k}\right)$ is a Banach space with respect to $\|$.$\| .$

The initial conditions of system (1) are given by

$$
\begin{aligned}
& x_{i}(s)=\varphi_{i}(s),-\tau \leq s \leq 0, \\
& \tau=\max _{1 \leq i, j \leq n}\left\{\tau_{i j}\right\}, 1 \leq i \leq n
\end{aligned}
$$

where

$$
\varphi(s)=\left(\varphi_{1}(s), \varphi_{2}(s), \cdots, \varphi_{n}(s)\right) \in C\left([-\tau, 0], R^{n}\right) .
$$

Le $x(t, \varphi)=\left(x_{1}(t, \varphi), x_{2}(t, \varphi), \cdots, x_{n}(t, \varphi)\right)^{\mathrm{T}}$ denotes any solution of the system (1) with initial value $\varphi \in C\left([-\tau, 0], R^{n}\right)$.

Definition 1. An solution $x(t, \varphi)$ of system (1) is said to be globally exponentially stable, for any solutions $x(t, \phi)$ of the system (1), if there exist positive constant $\lambda>0$ and $M>0$ such that

$$
\|x(t, \phi)-x(t, \varphi)\| \leq M\|\phi-\varphi\| \mathrm{e}^{-\lambda t}, t \geq 0
$$

Lemma 1. Under assumptions $\left(\mathrm{H}_{1}\right)-\left(\mathrm{H}_{3}\right)$, system (1) has a T-periodic solution which is globally exponentially stable, if the following conditions hold.

$\left(\mathrm{H}_{4}\right)$ Assume that there exist constants $\dot{\tau}_{i j}^{+}$such that $\dot{\tau}_{i j}(t) \leq \dot{\tau}_{i j}^{+}<1,1 \leq i, j \leq n$.
$\left(\mathrm{H}_{5}\right) \quad M_{1}=A_{1}-C_{1}$ is a nonsingular M-matrix, where

$$
\begin{aligned}
& A_{1}=\operatorname{diag}\left(\underline{\gamma}_{1}, \underline{\gamma}_{2}, \cdots, \underline{\gamma}_{n}\right), C_{1}=\left(c_{i j}\right)_{n \times n}, \\
& c_{i j}=\left(\bar{p}_{i j}+\bar{u}_{i j} \frac{1}{1-\dot{\tau}_{i j}^{+}}\right) L_{j} \rho_{j}
\end{aligned}
$$

Proof. If $v_{i j l}=0$, the model (2.1) in [14] reduces to the system (1), we know that Lemma 1 holds from Theorem 3.1 with $r=1$ in [14].

\section{Periodic Solutions of CGBAMNNs with Time Varying Delays}

Consider the following CGBAMNNs with time-varying delays:

$$
\begin{aligned}
\dot{x}_{i}(t)= & -a_{i}\left(x_{i}(t)\right)\left[b_{i}\left(t, x_{i}(t)\right)-\sum_{j=1}^{m} p_{i j}(t) f_{j}\left(\rho_{j} y_{j}(t)\right)\right. \\
& \left.-\sum_{j=1}^{m} u_{i j}(t) f_{j}\left(\rho_{j} y_{j}\left(t-\tau_{i j}(t)\right)\right)-I_{i}(t)\right] \\
\dot{y}_{j}(t)= & -c_{j}\left(y_{j}(t)\right)\left[d_{j}\left(t, y_{j}(t)\right)-\sum_{i=1}^{n} q_{j i}(t) g_{i}\left(\tilde{\rho}_{i} x_{i}(t)\right)\right. \\
& \left.-\sum_{i=1}^{n} v_{j i}(t) g_{i}\left(\tilde{\rho}_{i} x_{i}\left(t-\tilde{\tau}_{j i}(t)\right)\right)-J_{j}(t)\right]
\end{aligned}
$$

for $1 \leq i \leq n, 1 \leq j \leq m, t>0$ and $Z^{+}=\{1,2, \cdots\} . x_{i}(t)$ and $y_{j}(t)$ denote the state variables, $f_{j}(\cdot)$ and $g_{i}(\cdot)$ denote the signal functions, $I_{i}(t)$ and $J_{j}(t)$ denote inputs; $a_{i}(\cdot)$ and $c_{j}(\cdot)$ represent amplification functions; $b_{i}(t, \cdot)$ and $d_{j}(t, \cdot)$ are appropriately behaved functions; $p_{i j}(t), q_{j i}(t), u_{i j}(t)$ and $v_{j i}(t)$ are the connection weights and $\rho_{j}, \tilde{\rho}_{i}$ are positive constants, which correspond to the neuronal gains associated with the neuronal activations; Time delays $\tau_{i j}(t)$ and $\tilde{\tau}_{j i}(t)$ correspond to the finite speed of the axonal signal transmission at time $t$ and there exist constants $\tau_{i j}$ and $\tilde{\tau}_{j i}$ such that $0 \leq \tau_{i j} \leq \tau_{i j}, \quad 0 \leq \tilde{\tau}_{j i}(t) \leq \tilde{\tau}_{j i} ; \quad u_{i j}(t), \quad v_{j i}(t)$, $p_{i j}(t), \quad q_{j i}(t), I_{i}(t), \quad J_{j}(t), \tau_{i j}(t)$ and $\tilde{\tau}_{j i}(t)$ are all continuously periodic functions on $[0,+\infty)$ with common period $T>0$.

Throughout this paper, we assume for system (4) that $\left(\mathrm{H}_{6}\right)$ Amplification functions $a_{i}(\cdot)$ and $c_{j}(\cdot)$ are continuous and there exist positive constants $\underline{a}_{i}, \bar{a}_{i}$ and $\underline{c}_{j}, \bar{c}_{j}$ such that $\underline{a}_{i} \leq a_{i}\left(x_{i}(t)\right) \leq \bar{a}_{i}, \underline{c}_{j} \leq c_{j}\left(y_{j}(t)\right) \leq \bar{c}_{j}$, $1 \leq i \leq n, 1 \leq j \leq m$.

$\left(\mathrm{H}_{7}\right) b_{i}(t, \cdot), d_{j}(t, \cdot)$ are $T$-periodic about the first argument and there exist continuous $T$-periodic functions $\gamma_{i}(t)$ and $\delta_{j}(t)$ such that

$$
\begin{aligned}
& \frac{b_{i}(t, x)-b_{i}(t, y)}{x-y} \geq \gamma_{i}(t)>0, \frac{d_{j}(t, x)-d_{j}(t, y)}{x-y} \\
& \geq \delta_{j}(t)>0, \forall x \neq y \in R, 1 \leq i \leq n, 1 \leq j \leq m
\end{aligned}
$$

$\left(\mathrm{H}_{8}\right)$ For activation functions $f_{j}(\cdot)$ and $g_{i}(\cdot)$, there 
exist constants $L_{j}$ and $\tilde{L}_{i}$ such that

$$
\begin{aligned}
& L_{j}=\sup _{x \neq y}\left|\frac{f_{j}(x)-f_{j}(y)}{x-y}\right|, \tilde{L}_{i}=\sup _{x \neq y}\left|\frac{g_{i}(x)-g_{i}(y)}{x-y}\right|, \\
& \forall x \neq y \in \mathrm{R}, 1 \leq i \leq n, 1 \leq j \leq m
\end{aligned}
$$

The initial conditions of system (4) are given by

$$
\begin{aligned}
& x_{i}(s)=\varphi_{i}(s), y_{j}(s)=\phi_{j}(s),-\tilde{\tau} \leq s \leq 0, \\
& \tilde{\tau}=\max _{1 \leq i \leq n, 1 \leq j \leq m}\left\{\tau_{i j}, \tilde{\tau}_{j i}\right\}, 1 \leq i \leq n, 1 \leq j \leq m
\end{aligned}
$$

where

$$
\begin{aligned}
& (\varphi, \phi) \in C\left([-\tilde{\tau}, 0], R^{n+m}\right), \\
& \varphi(s)=\left(\varphi_{1}(s), \cdots, \varphi_{n}(s)\right), \phi(s)=\left(\phi_{1}(s), \cdots, \phi_{m}(s)\right) .
\end{aligned}
$$

Theorem 1. Under assumptions $\left(\mathrm{H}_{6}\right)-\left(\mathrm{H}_{10}\right)$, system (4) has a T-periodic solution which is globally exponentially stable, if the following condition holds.

$\left(\mathrm{H}_{9}\right)$ Assume that there exist constants $\dot{\tau}_{i j}^{+}$and $\dot{\tilde{\tau}}_{j i}^{+}$ such that $\dot{\tau}_{i j}(t) \leq \dot{\tau}_{i j}^{+}<1$ and $\dot{\tilde{\tau}}_{j i}(t) \leq \dot{\tilde{\tau}}_{j i}^{+}<1$ hold for $1 \leq i \leq n, 1 \leq j \leq m$.

$\left(\mathrm{H}_{10}\right)$ The following $M_{3}$ is a nonsingular M-matrix, and

$$
M_{3}=\left(\begin{array}{cc}
A_{3} & -C_{3} \\
-\tilde{C}_{3} & \tilde{A}_{3}
\end{array}\right)
$$

in which

$$
\begin{aligned}
& A_{3}=\operatorname{diag}\left(\left(\underline{\gamma}_{1}, \underline{\gamma}_{2}, \cdots, \underline{\gamma}_{n}\right), \tilde{A}_{3}=\operatorname{diag}\left(\underline{\delta}_{1}, \underline{\delta}_{2}, \cdots, \underline{\delta}_{m}\right)\right. \\
& \tilde{C}_{3}=\left(\tilde{c}_{j i}\right)_{m \times n}, \tilde{c}_{j i}=\left(\bar{q}_{j i}+\bar{v}_{j i} \frac{1}{1-\dot{\tilde{\tau}}_{j i}^{+}}\right) \tilde{L}_{i} \tilde{\rho}_{i}, \\
& C_{3}=\left(\hat{c}_{i j}\right)_{n \times m}, \hat{c}_{i j}=\left(\bar{p}_{i j}+\bar{u}_{i j} \frac{1}{1-\dot{\tau}_{i j}^{+}}\right) L_{j} \rho_{j}
\end{aligned}
$$

\section{Proof. Let}

$$
\begin{aligned}
& x_{n+j}(t)=y_{j}(t), a_{n+j}\left(t, x_{n+j}(t)\right)=c_{j}\left(t, y_{j}(t)\right), \\
& b_{n+j}\left(t, x_{n+j}(t)\right)=d_{j}\left(t, y_{j}(t)\right) \\
& p_{n+j, i}(t)=q_{j i}(t), p_{i, n+j}(t)=p_{i j}(t), \\
& u_{n+j, i}(t)=v_{j i}(t), u_{i, n+j}(t)=u_{i j}(t) \\
& S_{i}\left(x_{i}(t)\right)=g_{i}\left(x_{i}(t)\right), \\
& S_{n+j}\left(x_{n+j}(t)\right)=f_{j}\left(x_{j}(t)\right), \\
& \varphi_{n+j}(s)=\phi_{j}(s), I_{n+j}=J j(t) \\
& \tau_{n+j, i}(t)=\tilde{\tau}_{j i}(t), \tau_{i, j}(t)=\tilde{\tau}_{i, n+j}(t), \\
& \gamma_{n+j}(t)=\delta_{j}(t), \tilde{L}_{n+j}=L_{j}, \tilde{\rho}_{n+j}=\rho_{j},
\end{aligned}
$$

It follows that system (4) can be rewrote as

$$
\begin{aligned}
& \dot{x}_{i}(t)=-a_{i}\left(x_{i}(t)\right)\left[b_{i}\left(t, x_{i}(t)\right)\right. \\
& -\sum_{j=1}^{m} p_{i, n+j}(t) S_{n+j}\left(\tilde{\rho}_{n+j} x_{n+j}(t)\right) \\
& \left.-\sum_{j=1}^{m} u_{i, n+j}(t) S_{n+j}\left(\tilde{\rho}_{n+j} x_{n+j}\left(t-\tau_{i, n+j}(t)\right)\right)-I_{i}(t)\right], \\
& \dot{x}_{n+j}(t)=-a_{n+j}\left(x_{n+j}(t)\right)\left[b_{n+j}\left(t, x_{n+j}(t)\right)\right. \\
& -\sum_{i=1}^{n} p_{n+j, i}(t) S_{i}\left(\tilde{\rho}_{i} x_{i}(t)\right) \\
& \left.-\sum_{i=1}^{n} u_{n+j, i}(t) S_{i}\left(\tilde{\rho}_{i} x_{i}\left(t-\tau_{n+j, i}(t)\right)\right)-I_{n+j}(t)\right],
\end{aligned}
$$

for $1 \leq i \leq n, 1 \leq j \leq m$.

Initial conditions are given by

$$
x_{i}(s)=\varphi_{i}(s), s \in(-\tilde{\tau}, 0], i=1,2, \cdots,(n+m)
$$

Hence system (7) is a special case of system (1) in mathematical form in which there are $n+m$ neurons and connection weights $p_{i j}=u_{i j}=0$ for $1 \leq i, j \leq n$ and $n+1 \leq i, j \leq m+n$. Under conditions $\left(\mathrm{H}_{6}\right)-\left(\mathrm{H}_{10}\right)$, from Lemma 1, we obtain that system (7) has a $T$-periodic solution which is globally exponentially stable, if the following matrix $M^{\prime}$ is a $M$-matrix, and

$$
M^{\prime}=A^{\prime}-C^{\prime} \text {, }
$$

where

$$
\begin{aligned}
A^{\prime} & =\operatorname{diag}\left(\underline{\gamma}_{1}, \underline{\gamma}_{2}, \cdots, \underline{\gamma}_{n+m}\right) \\
C^{\prime} & =\left(\begin{array}{cccccc}
0 & \cdots & 0 & w_{1, n+1}^{\prime} & \cdots & w_{1, n+m}^{\prime} \\
\vdots & & \vdots & \vdots & & \vdots \\
0 & \cdots & 0 & w_{n, n+1}^{\prime} & \cdots & w_{n, n+m} \\
w_{n+1,1}^{\prime} & \cdots & w_{n+1, n}^{\prime} & 0 & \cdots & 0 \\
\vdots & & \vdots & \vdots & & \vdots \\
w_{n+m, 1}^{\prime} & \cdots & w_{n+m, n}^{\prime} & 0 & \cdots & 0
\end{array}\right)
\end{aligned}
$$

in which $w_{i j}^{\prime}=\left(\bar{p}_{i j}+\bar{u}_{i j} \frac{1}{1-\dot{\tau}_{i j}^{+}}\right) \tilde{\rho}_{j} \tilde{L}_{j}$.

Then, we know from (6) and (9) that Theorem 1 holds.

\section{An Example}

Consider the following CGBAMNNs with time delays:

$$
\begin{aligned}
\dot{x}_{1}(t) & =-\left(1+0.2 \cos \left(x_{1}(t)\right)\right) \\
\cdot & {\left[2.1 x_{1}(t)-2 \sin \pi t\left|y_{1}\left(t-\mathrm{e}^{-0.0001 \times t}\right)\right|-1\right], } \\
\dot{y}_{1}(t)= & -\left(1-0.2 \sin \left(y_{1}(t)\right)\right) \\
\cdot & {\left[2.1 y_{1}(t)-\cos \pi t\left|2 x_{1}(t-4)\right|-1\right], }
\end{aligned}
$$



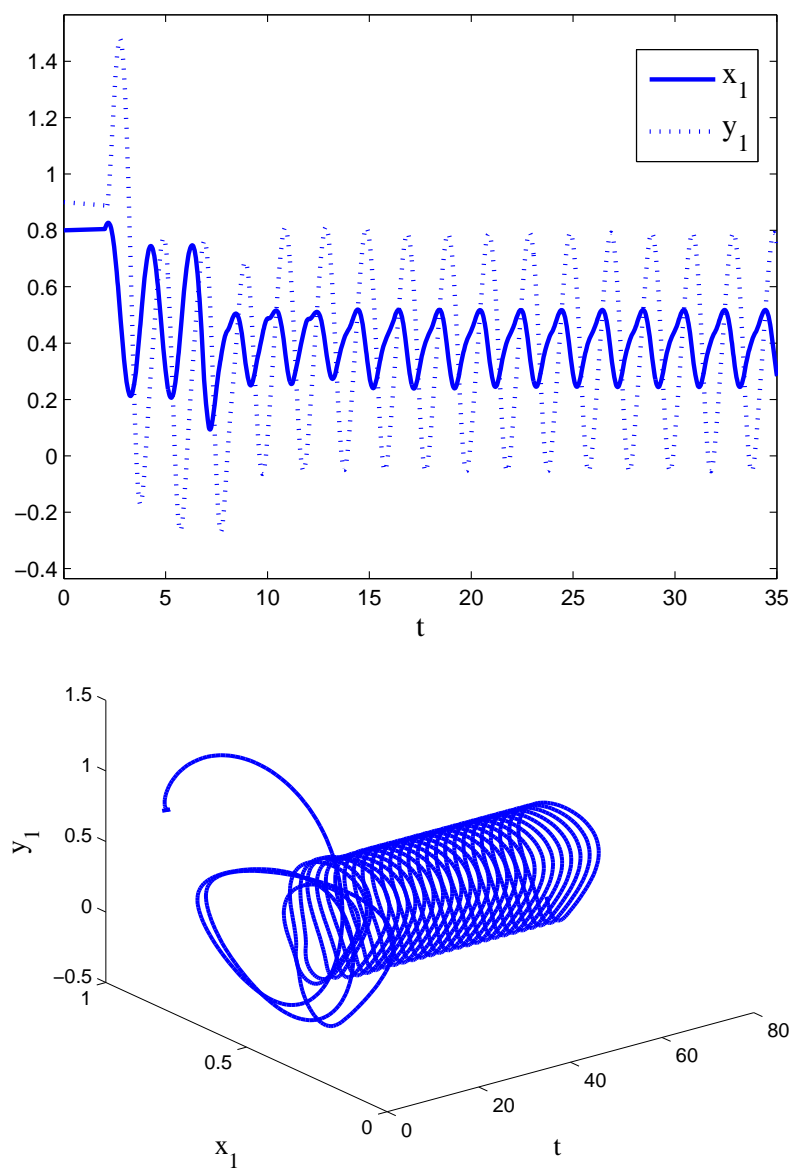

Figure 1. Time response of state variables $x_{1}, y_{1}$ and phase plot in space $\left(t, x_{1}, y_{1}\right)$ for system (10).

It is easy to verify system (10) satisfies $\left(\mathrm{H}_{6}\right)-\left(\mathrm{H}_{9}\right)$. In addition, system (10) satisfies $\left(\mathrm{H}_{10}\right)$ because

$$
M_{1}=\left(\begin{array}{cc}
2.1 & -1 \\
-2 & 2.1
\end{array}\right)
$$

is a nonsingular $M$-matrix. According to Theorem 1, system (10) has a 2-periodic solution which is globally exponentially stable. Figure 1 shows the dynamic behaveiors of system (10) with initial conditions $(0.8,0.9)$.

Remark 1 The results in $[3,15]$ have more restrictions than the results in this paper because conditions for the results in $[3,15]$ are relevant to amplification functions. In addition, in view of proof of Theorem 1, since CGBAMNNs with time-varying delays is a special case of CGNNs time-varying delays in form as BAM neural networks is a special case of Hopfield neural networks, many results of CGBAMNNs can be directly obtained from the ones of CGNNs, needing no repetitive discussions, which coincide with the conclusion in $[16,17]$.

\section{REFERENCES}

[1] S. Townley, S. Ilchmann, A. Weiss, et al., "Existence and
Learning of Oscillations in Recurrent Neural Networks," IEEE Transactions on Neural Networks, Vol. 11, No. 1, 2000, pp. 205-214. doi:10.1109/72.822523

[2] Z. Huang and Y. Xia, "Exponential Periodic Attractor of Impulsive BAM Networks with finite Distributed Delays," Chaos, Solitons \& Fractals, Vol. 39, No. 1, 2009, pp. 373-384. doi:10.1016/j.chaos.2007.04.014

[3] H. Xiang and J. Cao, "Exponential Stability of Periodic Solution to Cohen-Grossberg-Type BAM Networks with Time-Varying Delays," Neurocomputing, Vol. 72, No. 7-9, 2009, pp. 1702-1711. doi:10.1016/j.neucom.2008.07.006

[4] Y. Li, X. Chen and L. Zhao, "Stability and Existence of Periodic Solutions to Delayed Cohen-Grossberg BAM Neural Networks with Impulses on Timescales," Neurocomputing, Vol. 72, No. 7-9, 2009, pp. 1621-1630. doi:10.1016/j.neucom.2008.08.010

[5] Q. Song, J. Cao and Z. Zhao, "Periodic Solutions and Its Exponential Stability of Reaction-Diffusion Recurrent Neural Networks with Continuously Distributed Delays," Nonlinear Analysis: Real World Applications, Vol. 7, No. 1, 2006, pp. 65-80. doi:10.1016/j.nonrwa.2005.01.004

[6] T. Zhou, A. Chen and Y. Zhou, "Existence and Global Exponential Stability of Periodic Solution to BAM Neural Networks with Periodic Coefficient and Continuously Distributed Delays," Physics Letters A, Vol. 343, No. 5, 2005, pp. 336-350. doi:10.1016/j.physleta.2005.02.081

[7] J. Cao and J. Liang, "Boundedness and Stability for Cohen-Grossberg Neural Network with Time-Varying Delays," Journal of Mathematical Analysis and Applications, Vol. 296, No. 2, 2004, pp. 665-685. doi:10.1016/j.jmaa.2004.04.039

[8] H. Wang, X. Liao and C. Li, "Existence and Exponential Stability of Periodic Solution of BAM Neural Networks with Impulse and Time-Varying Delay," Chaos, Solitons \& Fractals, Vol. 33, No. 3, 2007, pp. 1028-1039. doi:10.1016/j.chaos.2006.01.112

[9] Q. Song and Z. Wang, "An Analysis on Existence and Global Exponential Stability of Periodic Solutions for BAM Neural Networks with Time-Varying Delays," Nonlinear Analysis: Real World Applications, Vol. 8, No. 4, 2007, pp. 1224-1234. doi:10.1016/j.nonrwa.2006.07.002

[10] Y. Li and J. Wang, "Analysis on the Global Exponential Stability and Existence of Periodic Solutions for NonAutonomous Hybrid BAM Neural Networks with Distributed Delays and Impulses," Computers \& Mathematics with Applications, Vol. 56, No. 9, 2008, pp. 22562267. doi:10.1016/j.camwa.2008.03.048

[11] X. Yang, "Existence and Global Exponential Stability of Periodic Solution for Cohen-Grossberg Shunting Inhibitory Cellular Neural Networks with Delays and Impulses," Neurocomputing, Vol. 72, No. 10-12, 2009, pp. 22192226. doi:10.1016/j.neucom.2009.01.003

[12] Q. Zhu, F. Liang and Q. Zhang, "Global Exponential Stability of Cohen-Grossberg Neural Networks with TimeVarying Delays and Impulses," Journal of Shanghai University, Vol. 13, No. 3, 2009, pp. 255-259.

doi:10.1007/s11741-009-0310-3 
[13] C. Li and S. Yang, "Existence and Attractivity of Periodic Solutions to Nonautonomous Cohen-Grossberg Neural Networks with Time Delays," Chaos, Solitons \& Fractals, Vol. 41, No. 3, 2009, pp. 1235-1244. doi:10.1016/j.chaos.2008.05.005

[14] Q. Liu, "Periodic Solutions of Higher-Order Cohen-Grossberg Neural Networks with Time-Varying Delays," Advances in Differential Equations and Control Process, Vol. 6, No. 1, 2010, pp. 1-14.

[15] A. Chen and Q. Gu, "Periodic Solution to BAM-Type Cohen-Grossberg Neural Network with Time-Varying Delays," Acta Mathematicae Applicatae Sinica, Vol. 27,
No. 3, 2011, pp. 427-442. doi:10.1007/s10255-011-0082-x

[16] J. Olivera, "Global Stability of a Cohen-Grossberg Neural Network with Both Time-Varying and Continuous Distributed Delays," Nonlinear Analysis: Real World Applications, Vol. 12, No. 5, 2011, pp. 2861-2870. doi:10.1016/j.nonrwa.2011.04.012

[17] Q. Liu and R. Xu, "Periodic Solutions of a Cohen-Grossberg-Type BAM Neural Networks with Distributed Delays and Impulses," Journal of Applied Mathematics, Vol. 2012, 2012, 17 p. 